\title{
NEW FRONTIERS IN THERMAL PLASMAS FROM SPACE TO NANOMATERIALS
}

\author{
MAHER I. BOULOS \\ President and CEO, Tekna Plasma Systems Inc. \\ Professor Émerit, University of Sherbrooke \\ Sherbrooke, Québec, Canada, J1L 2T9 \\ *Corresponding author. E-mail : Maher.Boulos@tekna.com
}

Received February 20, 2012

Thermal plasma technology has been at the center of major developments over the past century. It has found numerous applications ranging from aerospace materials testing to nanopowder synthesis and processing. In the present review highlights of principal breakthroughs in this field are presented with emphasis on an analysis of the basic phenomena involved, and the potential of the technology for industrial scale applications.

KEYWORDS : Thermal Plasmas, Aerospace Thermal Wind Tunnels. Induction Plasma, Plasma Spraying, Powder Purification. Powder Spherodization, Nanopowder Synthesis

\section{INTRODUCTION}

The plasma state is commonly referred to as the $4^{\text {th }}$ state of matter. The notion is based on the fact that if sufficient energy is supplied, solids can be melted to liquids, liquids can be vaporized to gases, and gases are then ionized to form plasma. Plasmas are partially ionized gases, containing ions, electrons, atoms and molecules, all in local electrical neutrality. The plasmas are observed under conditions ranging from high density and high energy thermonuclear fusion plasmas, to low temperature 'cold plasmas'. A representation of the different types of plasmas, on the electron temperature and electron density diagram, is given in Fig. 1. These corresponds a classification based on energy density and pressure diagram with the highest energy density plasmas known are those of the sun and of thermonuclear fusion. At lower energy densities we have glow discharges and florescent lamps, at low pressure, and thermal plasmas, arcs and radio frequency (rf) discharges, at atmospheric and higher pressures. At even lower energy densities one finds ionosphere plasmas, flames, and MHD generators.

In the present review we will limit our discussion to thermal plasmas as generated by direct current (dc) arcs or radio frequency (rf) inductively couples plasma discharges. Increasing attention has been given to these types of plasmas over the past four to five decades, initially for aerospace application involving materials testing for reentry simulation. More recently, attention has grown to include the use of thermal plasmas for materials processing such as plasma spray coating which is presently widely used in the aerospace, automotive, mining and bio-medical industries. The use of thermal plasma has more recently penetrated further in the electronic industry with the development of novel technologies, using thermal plasma sources, for the synthesis of nano-powders of metals and ceramics as well as ultra high purity materials in powder form.

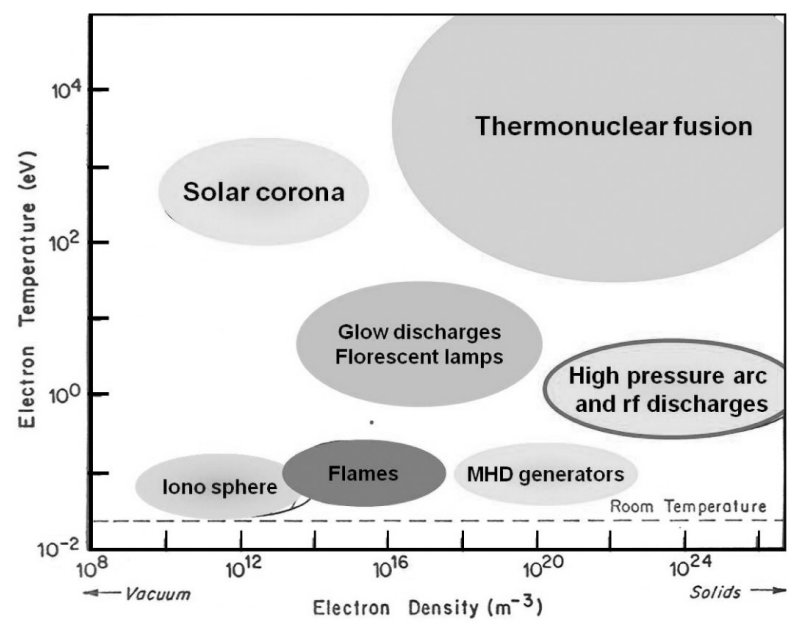

Fig. 1. Range of Plasma States on the Electron TemperatureElectron Density Diagram 


\section{THERMAL PLASMAS IN THE AEROSPACE INDUSTRY}

One of the early applications for thermal plasmas in the aerospace industry had as objective the simulation of the conditions meet during high and low altitude supersonic flight, and reentry of space vehicles. Numerous large scale wind tunnels were developed around the world for this purpose in the sixties and seventies. These were mostly based on dc arc plasma technology as identified in Fig. 2. Inductively Coupled Plasma (ICP) devices were used exclusively for the high altitude, low pressure testing. They have the main advantage of being able to be operated with air as the plasma gas with no potential metal-vapor contamination generated through electrode erosion which is the case in some of the high power dc plasma devices. DC devices on the other hand provide an excellent tool for high power and high energy density testing conditions which are mostly associated with lower altitude high speed flight conditions. A typical example of such devices developed at the Arnold Engineering Development Center in USA is shown in Fig. 3.

\section{THERMAL PLASMAS IN THE MATERIALS INDUSTRY}

Thermal plasmas are increasingly being recognized as a powerful tool for the synthesis and processing of a wide range of high added value materials. The principal applications that have been the subject of intensive R\&D work and industrial scale implementation are;

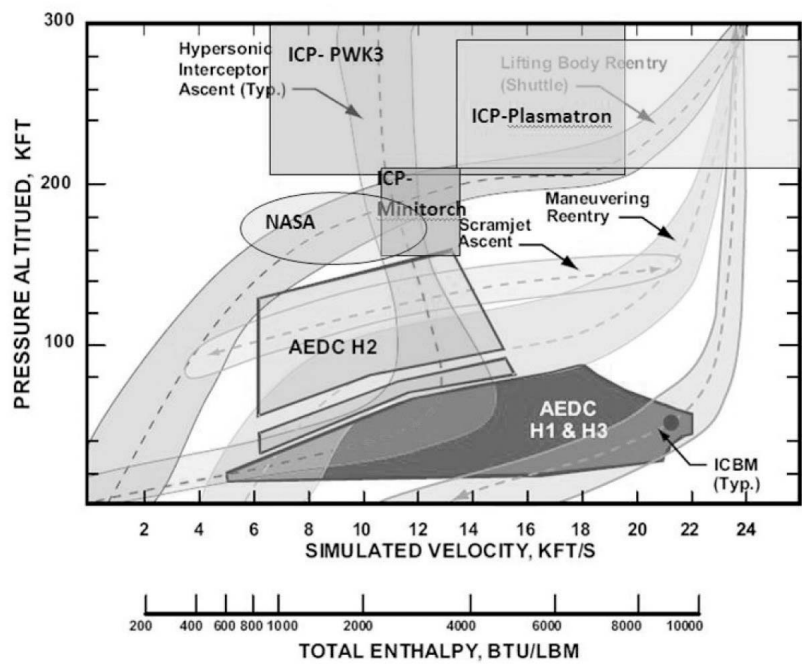

Fig. 2. Representation of the Conditions Generated by the Different Aerospace Testing Facilities as Function of Pressure Altitude and Simulated Velocity
- Plasma spraying of coatings and near net shaped parts.

- Plasma densification and purifications or powders. Often referred to as powder spherodization.

- Plasma synthesis of nanopowders.

In this section a brief description is given of each of these areas, highlighting technological potential and research needs for the further development and wider implementation of the technology.

\subsection{Plasma Spraying.}

In typical plasma spraying operation, schematically represented in Fig 4., the material to be deposited is injected into the plasma flow in the form of powder, which is heated and melted in-flight, before impacting on the surface of the substrate where it is flattened in the form of splats which, as shown in Fig. 5, accumulates on the top of each other thus forming the coating. The quality of the coating in terms of its apparent density and adhesion to the substrate is a direct function of the temperature and velocity of the individual droplets prior to their impact on the substrate. These depends, in-turn, on the spraying conditions such as the plasma power, plasma composition, chamber pressure, and the trajectory of the individual powder particles in the plasma flow.

The plasma spraying setups shown on the left hand side of Fig. 4 is based on the use of a dc plasma torch in which the plasma jet is generated by striking an arc between a central cathode and an annular anode. Both electrodes

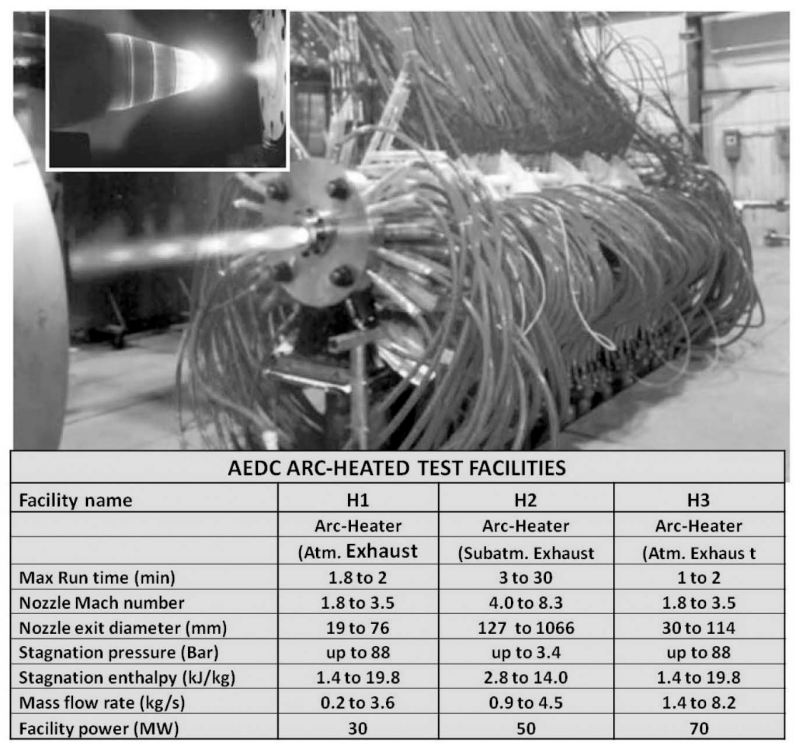

Fig. 3. Photograph of a Typical dc Plasma Torch and Corresponding Operating Conditions Developed by Arnold Engineering Development Center in the USA for Aerospace Applications. Torches $\mathrm{H} 1$ and $\mathrm{H} 3$ are Segmented while $\mathrm{H} 2$ is Huels. 
have to be water-cooled in order to protect them for the intense heat they receive from the arc. The plasma gas is injected in the annular gap between the two electrodes and serves to insure the continued motion of the arc root on the electrode surface before leaving the discharge cavity as a plasma jet with temperatures in the range of 10,000 to $12,000 \mathrm{~K}$, and velocities in the hundreds $\mathrm{m} / \mathrm{s}$. The powder to be used for the coating formation is injected externally to the torch at the exit level of the plasma jet using a powder-injection tube in which the powder is transported by a carrier gas and injected into the plasma flow. The injection tube is oriented in the radial toward the axis of the flow. The flow rate of the powder carrier gas is adjusted in such a way as to insure the penetration of the powder stream into the plasma flow in order to acquire an axial trajectory towards the substrate. In a typical dc plasma spraying system, the powder is in contact with the plasma flow for only a few milliseconds (1 to $2 \mathrm{~ms}$ ).

A schematic representation of an $\mathrm{rf}$ induction plasma spraying system is shown on the right hand side of Fig.4. In this case the plasma is generated through electromagnetic coupling of the input electrical energy into the discharge medium. The plasma torch is basically constructed of a non-conducting cylindrical plasma confinement tube made of silica or other ceramic materials and intensely water cooled. The electric energy is supplied to the torch through the use of a water-cooled induction coil, 4 to 6 turn, wound around the plasma confinement tube. As the coil is connected to the rf power source, an oscillating magnetic field is generated which will couple into the partially

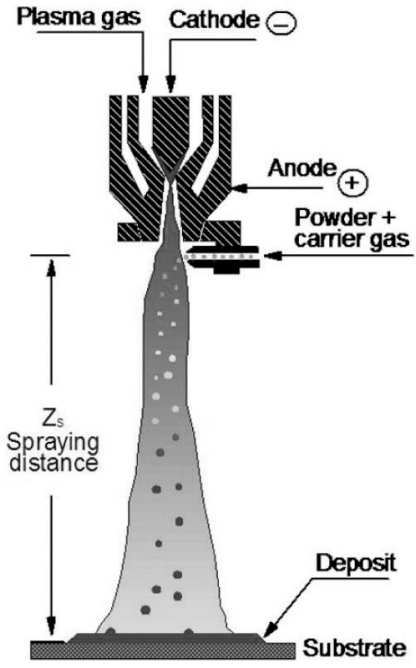

(a) d.c. plasma spraying

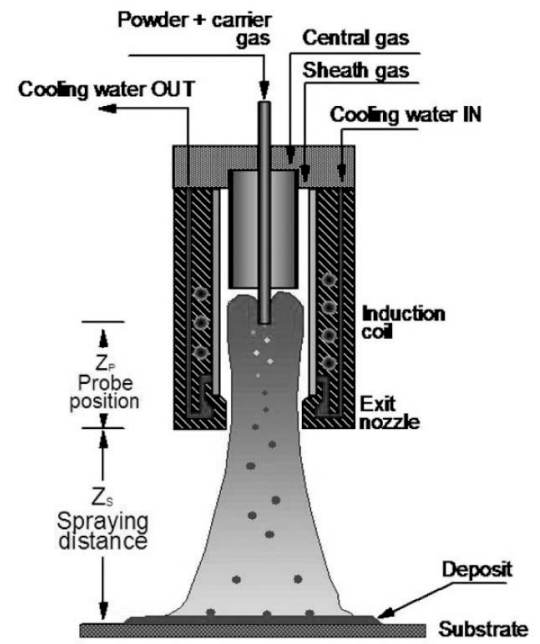

(b) r.f. induction plasma spraying

Fig. 4. Basic Setup used for dc and rf Induction Plasma Spraying

\section{The coating has a lamellar structure}
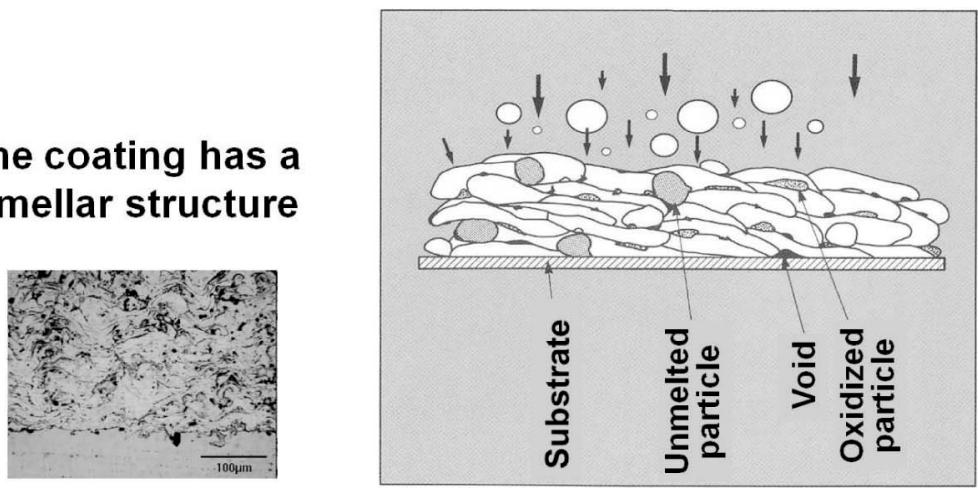

Fig. 5. Coating Buildup during Plasma Spraying Operation 
ionized gas load flowing within the discharge cavity, and providing for its ohmic heating in order to sustain the plasma. The plasma so generated is called 'inductively coupled plasma' or, in short, 'induction plasma'. Such plasma devoices have been built with power levels up to $200-300 \mathrm{~kW}$ or more and oscillator frequencies in the $\mathrm{MHz}$ range $(2-4 \mathrm{MHz})$. Because of the absence of any electrodes, as in the case of dc plasma sources, induction plasma torches can be used to operate with a wide range of plasma gases including, inert, oxidizing or reducing. The bulk plasma temperature is typically in the $10,000 \mathrm{~K}$ range and the plasma jet velocity at the exit of the torch nozzle is of the order of 10 to $30 \mathrm{~m} / \mathrm{s}$. higher velocities can be obtained when operating under reduced pressure conditions. In induction plasma spraying, the powder to be used in the coating formation is introduced, using a powder carrier gas, in the center of the discharge cavity through an axially oriented, water-cooled, powder injection probe. As the individual powder particles enter in contact with the plasma they assume an axial trajectory with a typical contact time between the plasma and the particles of the order to 10 to $30 \mathrm{~ms}$, During this time, the individual particles are heated, melted and accelerated toward the substrate on which they are deposited in essentialy the same way as with dc plasma spraying operations.

It is important to note that, over the past three to four decades, industrial applications of the wide range of thermal spray techniques developed, whether dc, rf plasma, wirearc, detonation guns or HVOF, have grown at a very fast rate in the aerospace industry where they are used for the coating of a large number of parts in jet engines and stationary turbines. Further penetration of the technology in a number of other applications in medical, electronic, mining, chemical and, more recently, in the automotive industry are reported as shown by the chart given in Fig 6 . On market segments for the thermal spray industry in 2002, after Ducos et al.

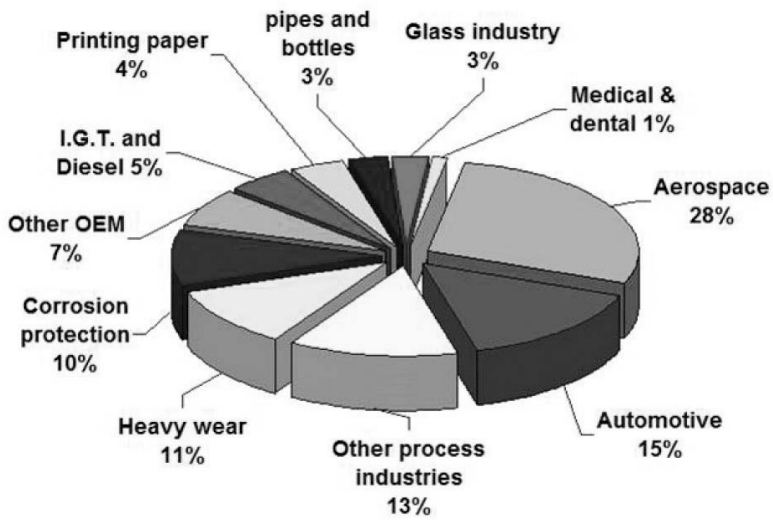

Fig. 6. Distribution of Market Segments for the Thermal Spray Industry by Application. After Ducos et al 2002

\subsection{Plasma Densification and Purifications of Powders}

The need for powder spherodization comes from very different industrial fields, e.g., from powder metallurgy to thermal spray applications. The most pressing need is for an industrial process to turn agglomerated powders, produced by spray drying or sintering techniques, and angular powders, produced by crushing of the process feed material, into spherical form powders. The transformation is to seek at least one of the following benefits resulting from the spherodization process.

a. Improves powder "flow-ability". Spherodization of particles provides a homogeneous, free-flowing character to the subject powder. This facilitates the powder handing and allows precise control of powder feed rates in a wide range of applications, including powder metallurgy and in various thermal spray processes. Hall flow test results have demonstrated that a material, which initially has poor flow-ability, could have its Hall flow time reduced by half as a result of its plasma spherodization treatment.

b. Increases powder packing density. Spherical particles provides denser packing of powders which increases overall bulk tap density.

c. Eliminates particle internal cavities and fractures. The melting of individual powder particles offers the means for eliminating the internal porosity of individual particles and consequently, increasing the particle hardness and overall powder bulk density.

d. Changes particle surface morphology. The macroscopic surface is made smoother. This effect benefits applications requiring lower inter-particle friction coefficients and low material contamination during pneumatic or other means of transport.

e. Enhances powder purity. The melting process can also be favorably used to enhance powder purity through the reactive vaporization of impurities. Through proper control of the plasma medium chemistry, induction plasma melting can provide significant increases in the purity of feed materials, by a factor of 10 to 100 , lowering impurities to the ppm range or less.

Spherical powders are also ideal for injection molding work, as well as applications for the thermal spraying of coatings or the forming of near net shape parts. For instance, in the field of thermal spraying, the quality of coatings (density, microstructure, etc.) can be significantly improved by the use of spherical, dense powder particles as the starting material. Metal Injection Molding (MIM) applications can also benefit from spherical powder's use for the resultant improvement of the flow-ability of the material.

While powder spherodization can be carried out using either dc or rf induction plasma, the induction plasma powder spherodization process, as represented schematically in Fig 7, has been widely accepted for its ability to insure 
the high quality and purity of the powders processed while achieving high throughput and economic viability. Basically the process is similar to induction plasma spraying, involving the in-flight heating and melting of individual particles of the powder feed material, followed by their gradual cooling and solidification under essentially "free fall" conditions. Depending on the particle size and apparent density of the treated powder, the time of flight is controlled such that the molten droplets have sufficient time for complete solidification before reaching the bottom of the primary processing chamber. Finer particles, which may still be entrained by the plasma gases, are recovered downstream of the primary processing chamber by means of an appropriate cyclone and/or filter collector.
Micrographs of molybdenum powders prior and after plasma processing in an induction plasma system are shown in Fig. 8 These show a considerable improvement of powder morphology associate with a significant increase of its tap density from an initial value of $2.3 \mathrm{~g} / \mathrm{cm}^{3}$ to $6.6 \mathrm{~g} / \mathrm{cm}^{3}$. The phenomena is best demonstrates On the left hand side of the figure where the same mass of Mo powder is show in two bottles prior and after plasma densification/ spherodization. The difference between the apparent volumes of the powder in each of these flasks is a direct indicator of the considerable increase of the apparent density of the powder through the plasma spherodization process. Thermal loading effects are one of the important phenomena to which special attention need to be given in

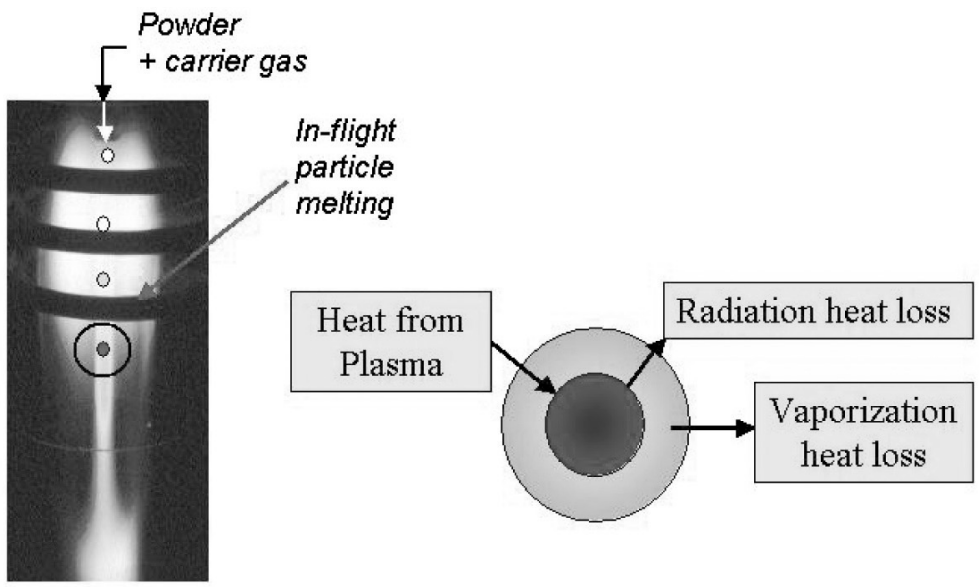

Fig. 7. Schematic Representation of the Basic Phenomena Involved in the Process of Spherodization Through the In-flight Heating and Melting of Individual Particles.

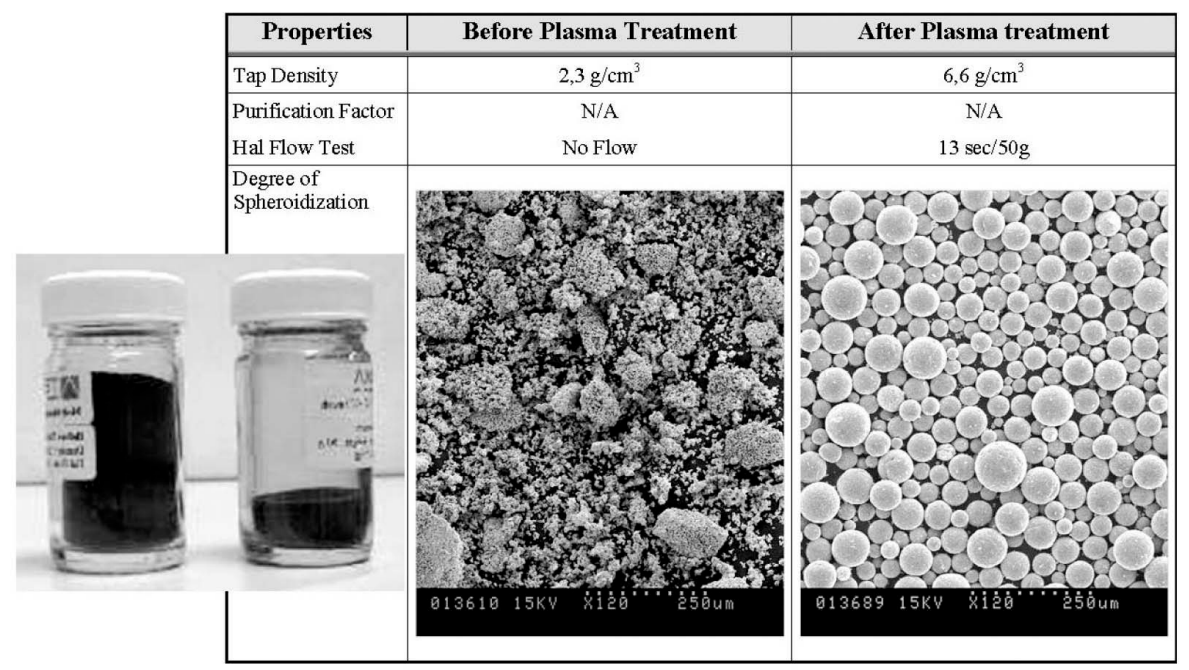

Fig. 8. Electron Micrographs of Molybdenum Powder Prior and After Treatment in an Induction Plasma 

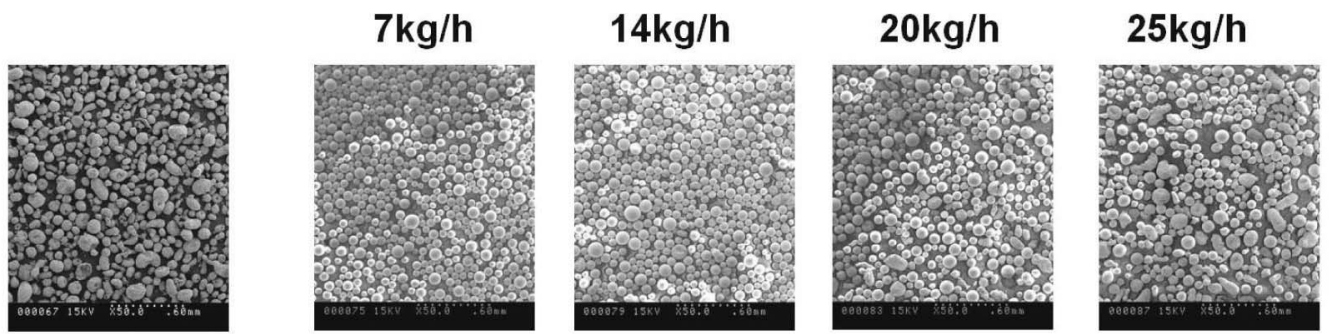

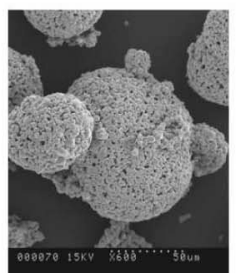

Tap Density : $\mathbf{3 . 2} \mathrm{g} / \mathrm{cc}$

Hall Flow $50 \mathrm{~g}: 36 \mathrm{~s}$

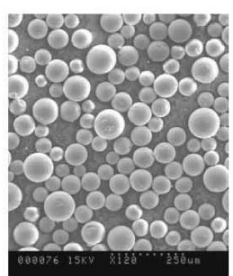

$6.35 \mathrm{~g} / \mathrm{cc}$

$13.0 \mathrm{~s}$

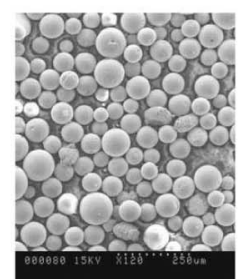

$6.25 \mathrm{~g} / \mathrm{cc}$

$14 \mathrm{~s}$

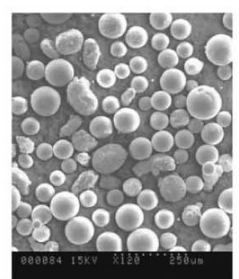

$5.8 \mathrm{~g} / \mathrm{cc}$

$16 \mathrm{~s}$

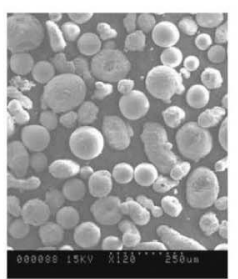

$5.0 \mathrm{~g} / \mathrm{cc}$

$19 \mathrm{~s}$

Fig. 9. Electron Micrographs of Induction Plasma Treated Molybdenum Powder under Different Powder Feed Rates. Plasma Composition $\mathrm{Ar} / \mathrm{H}_{2}$. Plasma Plate Power 100kW.

the industrial-scale processing of powders. With the increase of the powder throughput in the plasma, the local cooling of the plasma by the injected powder results in a decrease of the heat transfer potential between the plasma and the particles with a corresponding increase of the fraction of the particles that remains in the non-molten state. Accordingly the degree of melting that can be achieved under a given plasma condition at a given power density will depend directly on the powder feed rate injected into the plasma, which will in-turn has a direct effect on the economics of the process. A demonstration of this phenomenon is given in Fig 9 where electron micrographs of Molybdenum powders treated in a $100 \mathrm{~kW}, \mathrm{Ar} / \mathrm{H}_{2}$ plasma under different powder feed rates is shown. It can be observed that with the increase of the powder feed rate from 7 to $25 \mathrm{~kg} / \mathrm{h}$, the fraction of molten /spherodized powders drops with a corresponding drop of the associated tap density from 6.35 to $5.0 \mathrm{~g} / \mathrm{cm}^{3}$, and the increase of the Hal flow time from 13 to $19 \mathrm{~s} / 50 \mathrm{~g}$. Obviously the optimum processing conditions and associated powder feed rate would depend on the technical requirement of the final end use of the processed powder, and the process economics.

\subsection{Plasma Synthesis of Nanopowders}

The versatility of thermal plasma technology also offers the possibility to produce advanced powders at the nanometer scale. Indeed, the powder spherodization and the nanopowder synthesis can be performed on the same plasma unit, with only minor design modifications. The synthesis of nanopowders using thermal plasma technology is mostly carried out through the vaporization of the precursor material under plasma conditions followed by the rapid quench of the formed vapors under controlled conditions in order to condense the vapors in the form of an ultrafine aerosol with a controlled morphology, crystal structure and particle size distribution. Two techniques have been mostly used for feedstock vaporization. These are essentially base on either the in-flight vaporization of the feed material in the form of fine powder in a d.c. or r.f. inductively coupled discharge, or the vaporization of the precursor in the form of a molten metal pool in a transferred arc plasma furnace.

The induction plasma technology allows the processing of a wide variety of materials at a relatively high yield and affordable production cost. This convenient technology is well suitable to face the growing interest that arises not only from academic institutions and research centers, but more recently from industries in their search for a reliable and high capacity manufacturing technology. The two fundamental key features that make ICP technology attractive are the very high temperature processing and high quench rates. Since the temperature prevailing in the center of the discharge can reach $10,000^{\circ} \mathrm{C}$, reaction rates under such conditions are much faster than those found in conventional methods. On the other hand, the high quench rates at the exit of the reactor, which are typically $\sim 10^{5} \mathrm{~K} / \mathrm{s}$, prevents products dissociation and are responsible for particle condensation as an ultrafine powder with a typical particle size in the nanometer range (10-100 nm).

Various types of nanomaterial can be synthesized by carefully selecting the plasma gases and the appropriate reactant (gases, liquid solutions or suspensions and solids). 


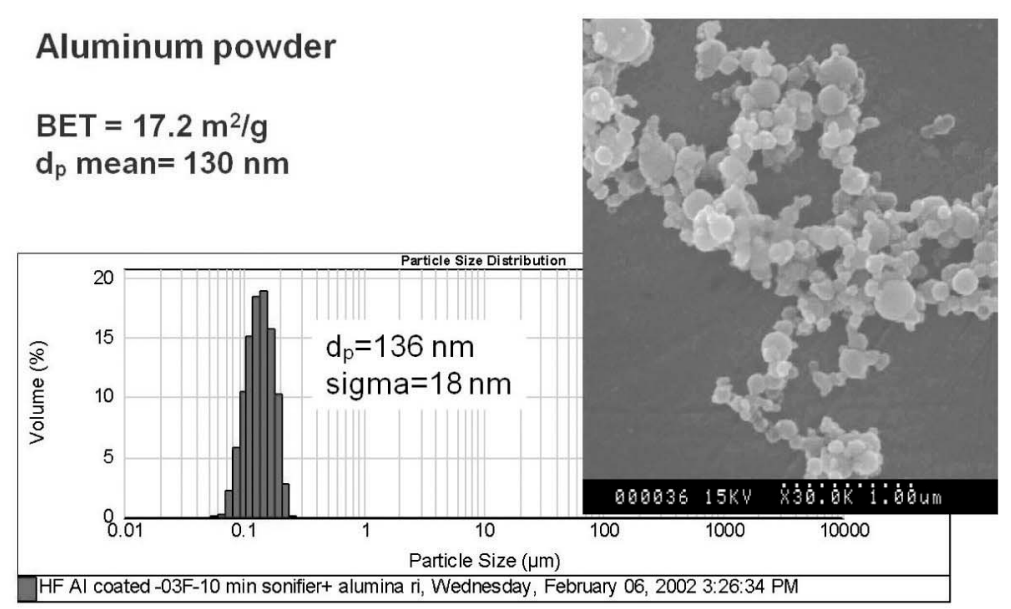

Fig. 10. Ultrafine Nanometric Aluminium Powder. BET $=17.2 \mathrm{~m}^{2} / \mathrm{g}, \mathrm{dp}=130 \mathrm{~nm}$.

For instance, reducing plasmas are commonly used to produce pure metallic nanopowders with oxygen-free surfaces, such as aluminum nanoparticles that are known to be extremely reactive in their non-passivated state. On the other hand, oxygen-rich plasmas are suitable for the synthesis of oxide materials, while inert plasmas are more appropriate in order to maintain the stoechiometry of the precursor. Typical electronmicrographs of aluminum nano-powders obtained with a laboratory scale induction plasma installation at $60 \mathrm{~kW}$ is given in Figs 10. The fact that the mean particle diameter of this powder, 130nm, based on BET specific surface measurements, is close to that obtained by laser scattering techniques, $136 \mathrm{~nm}$ is a clear indication of the non-agglomerated nature of the powder obtained in this case.

A wide range of nano-materials have been produced so far using ICP technology at the laboratory scale and pilot production level. These aim at improving the quality and particle size distribution of the powders obtained as well as on the associated yields. key $R \& D$ activities ongoing at Tekna Plasma Systems inc. focus on the improvement of ICP technology efficiency, especially in increasing the throughput on an industrial scale production level. It is important to underline that post-synthesis treatment of the powder might still be necessary for metallic powder passivation, Post synthesis classification of the powders, either using dry or wet classification techniques, seems to be also an inevitable step especially for the production of nanopowders with narrow particle size distributions.

\section{SUMMARY AND CONCLUSIONS}

A review is presented of available thermal plasma technologies that have been developed for a wide range of applications from aerospace materials testing to the synthesis of ultrafine nanopowders. Induction plasma technology has positioned itself for niche applications involving the in-flight heating melting, purification and vaporization of the feed material. Such techniques have been successfully used for the preparation of coating as well as spherical, dense high purity powders and nanopowders.

\section{REFERENCES}

[1 ] M.I. Boulos, P. Fauchais and E. Pfender, "Thermal Plasmas - Fundamentals and Applications", Plenum Press, Vol. 1 (1994).

[2] M.I. Boulos, "Radio frequency plasma developments, scaleup and industrial applications", High Temp. Chem. Processes 1, 401 (1992).

[ 3 ] U.S. Patent \# 5200595 and International PCT/CA92/ 00156.

[4] M. Rahmane, G. Soucy and M.I. Boulos, "Diffusion phenomena of a cold gas in thermal plasma stream", J. Plasma Chem. Plasma Proc. 16 169S (1996).

[ 5 ] M.I. Boulos and E. Pfender, "Material processing with thermal plasmas”, MRS Bulletin 8, 65 (1996).

[6] R. Ye et al., "In-flight spheroidization of alumina powders in $\mathrm{Ar}-\mathrm{H}_{2}$ and $\mathrm{Ar}-\mathrm{N}_{2}$ induction plasmas", Plasma Chem. Plasma Proc. 24555 (2004).

[7] Y.-L. Yi and T. Ishigaki, "Spheroidization of Titanium Carbide Powders by Induction Thermal Plasma Processing", J. Am. Ceram. Soc. 841929 (2001).

[ 8 ] N.M. Dignard and M.I. Boulos, "Ceramic and metallic powder spheroidization using induction plasma technology", Proceeding of Thermal Spray: a United Forum for Scientific and Technological Advances; Indianapolis, USA 419 (1998).

[9] I.A. Castillo and R.J. Munz, "Inductively coupled plasma synthesis of $\mathrm{CeO}_{2}$-based powders from liquid solutions for SOFC electrolytes", Plasma Chem. Plasma Proc. 25, 87 (2005).

[10] Y.-L. Yi and T. Ishigaki, "Controlled one-step synthesis of nanocrystalline anatase and rutile $\mathrm{TiO}_{2}$ powders by in- 
flight thermal plasma oxidation", J. Phys. Chem. B 108 15536 (2004).

[11] B.M. Goortani, N. Mendoza and P. Proulx, "Synthesis of $\mathrm{SiO}_{2}$ nanoparticles in $\mathrm{RF}$ plasma reactors: Effect of feed rate and quench gas injection", Int. J. Chem. Reactor Eng., 4 A33 (2005).
[12] J.W. Shin et al., "The influence of process parameters on precursor evaporation for alumina nanopowder synthesis in an inductively coupled rf thermal plasma", Plasma Sources Sci. Tech., 15, 441 (2006).

[13] F. Gitzhofer, "Induction plasma synthesis of ultrafine SiC", Pure Appl. Chem. 681113 (1996). 